\title{
Impulsive fractional boundary-value problems with fractional integral jump conditions
}

Chatthai Thaiprayoon ${ }^{1}$, Jessada Tariboon ${ }^{1 *}$ and Sotiris K Ntouyas ${ }^{2}$

\section{"Correspondence:}

jessadat@kmutnb.ac.th

1 Department of Mathematics,

Faculty of Applied Science, King

Mongkut's University of Technology

North Bangkok, Bangkok, Thailand

Full list of author information is

available at the end of the article

\begin{abstract}
In this paper we establish the existence and uniqueness of solutions for impulsive fractional boundary-value problems with fractional integral jump conditions. By using a variety of fixed-point theorems, some new existence and uniqueness results are obtained. Illustrative examples of our results are also presented.
\end{abstract}

MSC: 34A08; 34B37; 34B15; 34B10

Keywords: impulsive fractional differential equations; fractional integral jump conditions; fixed-point theorems

\section{Introduction}

In this paper, we investigate the following boundary-value problem for impulsive fractional differential equations with fractional integral jump conditions:

$$
\begin{cases}{ }^{c} D^{\alpha} x(t)=f(t, x(t)), & t \in[0, T] \backslash\left\{t_{1}, t_{2}, \ldots, t_{m}\right\}, \\ \Delta x\left(t_{k}\right)=J_{k}\left(\sum_{j=1}^{k} d_{k, j} I^{\beta_{k, j}} x\left(t_{j}^{-}\right)\right), & k=1,2, \ldots, m, \\ a x(0)+b x(T)=c, & \end{cases}
$$

where ${ }^{c} D^{\alpha}$ is the Caputo fractional derivative of order $\alpha, 0<\alpha<1, f:[0, T] \times \mathbb{R} \rightarrow \mathbb{R}$ is a continuous function, $J_{k}: \mathbb{R} \rightarrow \mathbb{R}, \Delta x\left(t_{k}\right)=x\left(t_{k}^{+}\right)-x\left(t_{k}^{-}\right)$with $x\left(t_{k}^{+}\right)=\lim _{h \rightarrow 0^{+}} x\left(t_{k}+h\right)$, $x\left(t_{k}^{-}\right)=\lim _{h \rightarrow 0^{-}} x\left(t_{k}+h\right), d_{k, j}$ are constants, $I^{\beta_{k, j}}$ is the Riemann-Liouville fractional integral of order $\beta_{k, j}>0$ for $j=1,2 \ldots, k$ and $k=1,2, \ldots, m, 0=t_{0}<t_{1}<\cdots<t_{m}<t_{m+1}=T, a, b, c$ are given constants such that $a+b \neq 0$.

The integral jump conditions are very general and include many conditions as special cases. In particular, if $d_{k, j}=d_{j}$ and $\beta_{k, j}=\beta_{j}$, then the impulsive fractional integral of equation (1.1) reduces to

$$
\begin{aligned}
\Delta x\left(t_{k}\right)= & J_{k}\left(d_{1} \int_{0}^{t_{1}} \frac{(t-s)^{\beta_{1}-1}}{\Gamma\left(\beta_{1}\right)} x(s) d s+d_{2} \int_{0}^{t_{2}} \frac{(t-s)^{\beta_{2}-1}}{\Gamma\left(\beta_{2}\right)} x(s) d s+\cdots\right. \\
& \left.+d_{k} \int_{0}^{t_{k}} \frac{(t-s)^{\beta_{k}-1}}{\Gamma\left(\beta_{k}\right)} x(s) d s\right) .
\end{aligned}
$$

Recently, much attention has been paid to the existence of solutions for fractional differential equations due to its wide application in engineering, economics and other fields.

(02014 Thaiprayoon et al.; licensee Springer. This is an Open Access article distributed under the terms of the Creative Commons Attribution License (http://creativecommons.org/licenses/by/2.0), which permits unrestricted use, distribution, and reproduction in any medium, provided the original work is properly cited. 
A variety of results on initial- and boundary-value problems of fractional differential equations and inclusions can easily be found in the literature on the topic. For some recent results, we can refer to [1-13] and references cited therein.

On the other hand, integer order impulsive differential equations have become important in recent years as mathematical models of phenomena in both the physical and social sciences. There has a significant development in impulsive theory especially in the area of impulsive differential equations with fixed moments, see for instance [14-24].

In this paper we prove some new existence and uniqueness results by using a variety of fixed-point theorems. In Theorem 3.1 we prove an existence and uniqueness result by using Banach's contraction principle, in Theorem 3.2 we prove an existence and uniqueness result by using Banach's contraction principle and Hölder's inequality, in Theorem 3.3 we prove the existence of a solution by using Krasnoselskii's fixed-point theorem, while in Theorem 3.4 we prove the existence of a solution via Leray-Schauder's nonlinear alternative. Leray-Schauder's degree theory is used in proving the existence result in Theorem 3.5.

The rest of the paper is organized as follows: In Section 2 we recall some preliminaries and present a basic lemma which is used to convert the impulsive fractional boundaryvalue problem (1.1) into an equivalent integral equation. The main results are presented in Section 3, while illustrative examples are contained in Section 4.

\section{Preliminaries}

Let $\mathrm{PC}([0, T], \mathbb{R})=\left\{x:[0, T] \rightarrow \mathbb{R}: x(t)\right.$ is continuous everywhere except for some $t_{k}$ at which $x\left(t_{k}^{+}\right)$and $x\left(t_{k}^{-}\right)$exist and $\left.x\left(t_{k}^{-}\right)=x\left(t_{k}\right), k=1,2, \ldots, m\right\} . \operatorname{PC}([0, T], \mathbb{R})$ is a Banach space endowed with the norm defined by $\|x\|=\sup _{t \in[0, T]}|x(t)|$. Next, we introduce some notations, definitions of fractional calculus [25-27], and we present a preliminary result needed in our proofs later.

Definition 2.1 The Riemann-Liouville fractional integral of order $\alpha>0$ of a function $g \in$ $L^{1}((0, T), \mathbb{R})$ is defined by

$$
I^{\alpha} g(t)=\int_{0}^{t} \frac{(t-s)^{\alpha-1}}{\Gamma(\alpha)} g(s) d s,
$$

where $\Gamma$ is the Gamma function.

Definition 2.2 The Riemann-Liouville fractional derivative of order $\alpha>0$ of a continuous function $g:(0, \infty) \rightarrow \mathbb{R}$ is defined by

$$
D^{\alpha} g(t)=\frac{1}{\Gamma(n-\alpha)}\left(\frac{d}{d t}\right)^{n} \int_{0}^{t} \frac{g(s)}{(t-s)^{\alpha-n+1}} d s,
$$

where $n=[\alpha]+1,[\alpha]$ denotes the integral part of real number $\alpha$, provided the right-hand side is point-wise defined on $(0, \infty)$.

Definition 2.3 For a continuous function $g:(0, \infty) \rightarrow \mathbb{R}$, the Caputo derivative of fractional order $\alpha$ is defined as

$$
{ }^{c} D^{\alpha} g(t)=\frac{1}{\Gamma(n-\alpha)} \int_{0}^{t}(t-s)^{n-\alpha-1} g^{(n)}(s) d s,
$$

where $n=[\alpha]+1,[\alpha]$ denotes the integral part of real number $\alpha$, provided $g^{(n)}(t)$ exists. 
Lemma 2.1 ([28]) Let $\alpha \in(0,1)$ and $h:[0, T] \rightarrow \mathbb{R}$ be continuous. A function $x \in$ $C([0, T], \mathbb{R})$ is a solution of the fractional Cauchy problem

$$
\begin{cases}{ }^{c} D^{\alpha} x(t)=h(t), & t \in[0, T], \\ x(\eta)=x_{0}, & \eta>0,\end{cases}
$$

if and only if $x$ is a solution of the following integral equation:

$$
\begin{aligned}
x(t)= & x_{0}-\frac{1}{\Gamma(\alpha)} \int_{0}^{\eta}(\eta-s)^{\alpha-1} h(s) d s \\
& +\frac{1}{\Gamma(\alpha)} \int_{0}^{t}(t-s)^{\alpha-1} h(s) d s .
\end{aligned}
$$

Lemma 2.2 Let $0<\alpha<1$ and $a+b \neq 0$. The unique solution of the impulsive fractional boundary-value problem (1.1) is given by

$$
\begin{aligned}
x(t)= & \frac{c}{a+b}-\frac{b}{a+b}\left[\sum_{i=1}^{m} J_{i}\left(\sum_{j=1}^{i} d_{i, j} I^{\beta_{i, j}} x\left(t_{j}^{-}\right)\right)+\frac{1}{\Gamma(\alpha)} \int_{0}^{T}(T-s)^{\alpha-1} f(s, x(s)) d s\right] \\
& +\sum_{i=1}^{k} J_{i}\left(\sum_{j=1}^{i} d_{i, j} I^{\beta_{i, j}} x\left(t_{j}^{-}\right)\right)+\frac{1}{\Gamma(\alpha)} \int_{0}^{t}(t-s)^{\alpha-1} f(s, x(s)) d s .
\end{aligned}
$$

Proof For $t \in\left[t_{0}, t_{1}\right]$, Riemann-Liouville fractional integrating of order $\alpha$, from 0 to $t$, for the first equation of (1.1), we have

$$
x(t)=x(0)+\frac{1}{\Gamma(\alpha)} \int_{0}^{t}(t-s)^{\alpha-1} f(s, x(s)) d s .
$$

Substituting $t=t_{1}$ into (2.2), we get

$$
x\left(t_{1}\right)=x(0)+\frac{1}{\Gamma(\alpha)} \int_{0}^{t_{1}}\left(t_{1}-s\right)^{\alpha-1} f(s, x(s)) d s .
$$

For $t \in\left(t_{1}, t_{2}\right]$, by Lemma 2.1 with the second equation of (1.1), we obtain

$$
\begin{aligned}
x(t)= & x\left(t_{1}^{+}\right)-\frac{1}{\Gamma(\alpha)} \int_{0}^{t_{1}}\left(t_{1}-s\right)^{\alpha-1} f(s, x(s)) d s \\
& +\frac{1}{\Gamma(\alpha)} \int_{0}^{t}(t-s)^{\alpha-1} f(s, x(s)) d s \\
= & x\left(t_{1}\right)+J_{1}\left(\sum_{j=1}^{1} d_{1, j} I^{\beta_{1, j}} x\left(t_{j}^{-}\right)\right)-\frac{1}{\Gamma(\alpha)} \int_{0}^{t_{1}}\left(t_{1}-s\right)^{\alpha-1} f(s, x(s)) d s \\
& +\frac{1}{\Gamma(\alpha)} \int_{0}^{t}(t-s)^{\alpha-1} f(s, x(s)) d s \\
= & x(0)+J_{1}\left(\sum_{j=1}^{1} d_{1, j} I^{\beta_{1, j}} x\left(t_{j}^{-}\right)\right)+\frac{1}{\Gamma(\alpha)} \int_{0}^{t}(t-s)^{\alpha-1} f(s, x(s)) d s .
\end{aligned}
$$


If $t \in\left(t_{2}, t_{3}\right]$ then again from Lemma 2.1, we have

$$
\begin{aligned}
x(t) & =x\left(t_{2}^{+}\right)-\frac{1}{\Gamma(\alpha)} \int_{0}^{t_{2}}\left(t_{2}-s\right)^{\alpha-1} f(s, x(s)) d s+\frac{1}{\Gamma(\alpha)} \int_{0}^{t}(t-s)^{\alpha-1} f(s, x(s)) d s \\
& =x(0)+\sum_{i=1}^{2} J_{i}\left(\sum_{j=1}^{i} d_{i, j} I^{\beta_{i, j}} x\left(t_{j}^{-}\right)\right)+\frac{1}{\Gamma(\alpha)} \int_{0}^{t}(t-s)^{\alpha-1} f(s, x(s)) d s .
\end{aligned}
$$

If $t \in\left(t_{k}, t_{k+1}\right]$ then again from Lemma 2.1, we get

$$
x(t)=x(0)+\sum_{i=1}^{k} J_{i}\left(\sum_{j=1}^{i} d_{i, j} I^{\beta_{i, j}} x\left(t_{j}^{-}\right)\right)+\frac{1}{\Gamma(\alpha)} \int_{0}^{t}(t-s)^{\alpha-1} f(s, x(s)) d s .
$$

In particular, for $t=T$, we have

$$
x(T)=x(0)+\sum_{i=1}^{m} J_{i}\left(\sum_{j=1}^{i} d_{i, j} I^{\beta_{i, j}} x\left(t_{j}^{-}\right)\right)+\frac{1}{\Gamma(\alpha)} \int_{0}^{T}(T-s)^{\alpha-1} f(s, x(s)) d s
$$

From the third equation of (1.1) and (2.3), we get

$$
x(0)=\frac{c}{a+b}-\frac{b}{a+b}\left[\sum_{i=1}^{m} J_{i}\left(\sum_{j=1}^{i} d_{i, j} I^{\beta_{i, j}} x\left(t_{j}^{-}\right)\right)+\frac{1}{\Gamma(\alpha)} \int_{0}^{T}(T-s)^{\alpha-1} f(s, x(s)) d s\right] .
$$

Therefore, we have

$$
\begin{aligned}
x(t)= & \frac{c}{a+b}-\frac{b}{a+b}\left[\sum_{i=1}^{m} J_{i}\left(\sum_{j=1}^{i} d_{i, j} I^{\beta_{i, j}} x\left(t_{j}^{-}\right)\right)+\frac{1}{\Gamma(\alpha)} \int_{0}^{T}(T-s)^{\alpha-1} f(s, x(s)) d s\right] \\
& +\sum_{i=1}^{k} J_{i}\left(\sum_{j=1}^{i} d_{i, j} I^{\beta_{i, j}} x\left(t_{j}^{-}\right)\right)+\frac{1}{\Gamma(\alpha)} \int_{0}^{t}(t-s)^{\alpha-1} f(s, x(s)) d s .
\end{aligned}
$$

This completes the proof.

As in Lemma 2.2, we define an operator $A: \mathrm{PC}([0, T], \mathbb{R}) \rightarrow \mathrm{PC}([0, T], \mathbb{R})$ by

$$
\begin{aligned}
(A x)(t)= & \frac{c}{a+b}-\frac{b}{a+b}\left[\sum_{i=1}^{m} J_{i}\left(\sum_{j=1}^{i} d_{i, j} I^{\beta_{i, j}} x\left(t_{j}^{-}\right)\right)+\frac{1}{\Gamma(\alpha)} \int_{0}^{T}(T-s)^{\alpha-1} f(s, x(s)) d s\right] \\
& +\sum_{i=1}^{k} J_{i}\left(\sum_{j=1}^{i} d_{i, j} I^{\beta_{i, j}} x\left(t_{j}^{-}\right)\right)+\frac{1}{\Gamma(\alpha)} \int_{0}^{t}(t-s)^{\alpha-1} f(s, x(s)) d s
\end{aligned}
$$

with $a+b \neq 0$. It should be noticed that problem (1.1) has solutions if and only if the operator $A$ has fixed points.

\section{Main results}

We are in a position to establish our main results. In the following subsections we prove existence as well as existence and uniqueness results for the impulsive fractional BVP (1.1) by using a variety of fixed-point theorems. 


\subsection{Existence and uniqueness results via Banach's fixed-point theorem}

In this subsection we give first an existence and uniqueness result for the impulsive fractional BVP (1.1) by using Banach's fixed-point theorem.

For convenience, we set

$$
\begin{aligned}
& \Omega=\frac{|a+b|+|b|}{|a+b| \Gamma(\alpha+1)} T^{\alpha}, \\
& \Phi=\left(\frac{|a+b|+|b|}{|a+b|}\right) \sum_{i=1}^{m} \sum_{j=1}^{i} \frac{\left|d_{i, j}\right| t_{j}^{\beta_{i, j}}}{\Gamma\left(\beta_{i, j}+1\right)}, \\
& \Psi=\frac{(|a+b|+|b|) m N+|c|}{|a+b|} .
\end{aligned}
$$

Theorem 3.1 Assume the following.

$\left(\mathrm{H}_{1}\right)$ There exists a constant $L_{1}>0$ such that $|f(t, x)-f(t, y)| \leq L_{1}|x-y|$, for each $t \in[0, T]$ and $x, y \in \mathbb{R}$.

$\left(\mathrm{H}_{2}\right)$ There exists a constant $L_{2}>0$ such that $\left|J_{k}(x)-J_{k}(y)\right| \leq L_{2}|x-y|$ for each $x, y \in \mathbb{R}$, $k=1,2, \ldots, m$.

If

$$
\Lambda:=L_{1} \Omega+L_{2} \Phi \leq \delta \leq \varepsilon<1,
$$

then impulsive fractional boundary-value problem (1.1) has a unique solution in $[0, T]$.

Proof We transform the problem (1.1) into a fixed-point problem, $x=A x$, where the operator $A$ is defined by equation (2.4). Using Banach's contraction principle, we shall show that $A$ has a fixed point.

Setting $\sup _{t \in[0, T]}|f(t, 0)|=M<\infty, \sup \left\{\left|J_{k}(0)\right| ; k=1,2, \ldots, m\right\}=N<\infty$ and choosing $r \geq$ $\frac{1}{1-\varepsilon}(M \Omega+\Psi)$, we show that $A B_{r} \subset B_{r}$, where $B_{r}=\{x \in \mathrm{PC}([0, T], \mathbb{R}):\|x\| \leq r\}$. For $x \in B_{r}$, we have

$$
\begin{aligned}
\|A x\| \leq & \sup _{t \in[0, T]}\left\{\frac{|b|}{|a+b|}\left[\sum_{i=1}^{m}\left|J_{i}\left(\sum_{j=1}^{i} d_{i, j} I^{\beta_{i, j}} x\left(t_{j}^{-}\right)\right)\right|+\frac{1}{\Gamma(\alpha)} \int_{0}^{T}(T-s)^{\alpha-1}|f(s, x(s))| d s\right]\right. \\
& \left.+\sum_{i=1}^{k}\left|J_{i}\left(\sum_{j=1}^{i} d_{i, j} I^{\beta_{i, j}} x\left(t_{j}^{-}\right)\right)\right|+\frac{1}{\Gamma(\alpha)} \int_{0}^{t}(t-s)^{\alpha-1}|f(s, x(s))| d s+\frac{|c|}{|a+b|}\right\} \\
\leq & \frac{|a+b|+|b|}{|a+b|}\left\{\sum_{i=1}^{m}\left[\left|J_{i}\left(\sum_{j=1}^{i} d_{i, j} I^{\beta_{i, j}} x\left(t_{j}^{-}\right)\right)-J_{i}(0)\right|+\left|J_{i}(0)\right|\right]\right. \\
& \left.+\frac{1}{\Gamma(\alpha)} \int_{0}^{T}(T-s)^{\alpha-1}(|f(s, x(s))-f(s, 0)|+|f(s, 0)|) d s\right\}+\frac{|c|}{|a+b|} \\
\leq & \frac{|a+b|+|b|}{|a+b|}\left\{\sum_{i=1}^{m}\left[L_{2}\left|\sum_{j=1}^{i} d_{i, j} I^{\beta_{i, j}} x\left(t_{j}^{-}\right)\right|+N\right]\right. \\
& \left.+\frac{L_{1} r+M}{\Gamma(\alpha)} \int_{0}^{T}(T-s)^{\alpha-1} d s\right\}+\frac{|c|}{|a+b|}
\end{aligned}
$$




$$
\begin{aligned}
& \leq \frac{|a+b|+|b|}{|a+b|}\left\{\sum_{i=1}^{m}\left[L_{2} r \sum_{j=1}^{i} \frac{\left|d_{i, j}\right| t_{j}^{\beta_{i, j}}}{\Gamma\left(\beta_{i, j}+1\right)}+N\right]+\frac{\left(L_{1} r+M\right) T^{\alpha}}{\Gamma(\alpha+1)}\right\}+\frac{|c|}{|a+b|} \\
& =\left(L_{1} r+M\right) \Omega+L_{2} r \Phi+\Psi \\
& \leq(\delta+1-\varepsilon) r \leq r,
\end{aligned}
$$

which proves that $A B_{r} \subset B_{r}$.

Now let $x, y \in \operatorname{PC}([0, T], \mathbb{R})$. Then, for $t \in[0, T]$, we have

$$
\begin{aligned}
|(A x)(t)-(A y)(t)| \leq & \sup _{t \in[0, T]}\left\{\frac { | b | } { | a + b | } \left[\sum_{i=1}^{m}\left|J_{i}\left(\sum_{j=1}^{i} d_{i, j} I^{\beta_{i, j}} x\left(t_{j}^{-}\right)\right)-J_{i}\left(\sum_{j=1}^{i} d_{i, j} I^{\beta_{i, j}} y\left(t_{j}^{-}\right)\right)\right|\right.\right. \\
& \left.+\frac{1}{\Gamma(\alpha)} \int_{0}^{T}(T-s)^{\alpha-1}|f(s, x(s))-f(s, y(s))| d s\right] \\
& +\sum_{i=1}^{k}\left|J_{i}\left(\sum_{j=1}^{i} d_{i, j} I^{\beta_{i, j}} x\left(t_{j}^{-}\right)\right)-J_{i}\left(\sum_{j=1}^{i} d_{i, j} j^{\beta_{i, j}} y\left(t_{j}^{-}\right)\right)\right| \\
& \left.+\frac{1}{\Gamma(\alpha)} \int_{0}^{t}(t-s)^{\alpha-1}|f(s, x(s))-f(s, y(s))| d s\right\} \\
\leq & \frac{|a+b|+|b|}{|a+b|}\left[L_{2}\|x-y\| \sum_{i=1}^{m} \sum_{j=1}^{i} \frac{\left|d_{i, j}\right|}{\Gamma\left(\beta_{i, j}\right)} \int_{0}^{t_{j}}\left(t_{j}-s\right)^{\beta_{i, j}-1} d s\right. \\
& \left.+\frac{L_{1}\|x-y\|}{\Gamma(\alpha)} \int_{0}^{T}(T-s)^{\alpha-1} d s\right] \\
= & L_{1} \Omega\|x-y\|+L_{2} \Phi\|x-y\| .
\end{aligned}
$$

Therefore,

$$
\|A u-A v\| \leq \Lambda\|u-v\| .
$$

As follows from equation (3.4), $A$ is a contraction. As a consequence of Banach's fixedpoint theorem, we have $A$ has a fixed point which is a unique solution of the impulsive fractional boundary-value problem (1.1). This completes the proof.

Now we give another existence and uniqueness result for impulsive fractional BVP (1.1) by using Banach's fixed-point theorem and Hölder's inequality. In addition, for $\sigma \in(0,1)$, we set

$$
\begin{aligned}
& \Omega^{*}=\left(\frac{|a+b|+|b|}{|a+b| \Gamma(\alpha)}\right)\left(\frac{1-\gamma}{\alpha-\gamma}\right)^{1-\gamma} T^{\alpha-\gamma} \\
& \Phi^{*}=\left(\frac{|a+b|+|b|}{|a+b|}\right)\left(\sum_{i=1}^{m}\left(\sum_{j=1}^{i} \frac{\left|d_{i, j}\right| t_{j}^{\beta_{i j}}}{\Gamma\left(\beta_{i, j}+1\right)}\right)^{\frac{1}{1-\sigma}}\right)^{1-\sigma}, \\
& \eta^{*}=\left(\sum_{i=1}^{m}\left(\eta_{i}\right)^{\frac{1}{\sigma}}\right)^{\sigma} .
\end{aligned}
$$


Theorem 3.2 Assume that the following conditions hold:

$\left(\mathrm{H}_{3}\right)|f(t, x)-f(t, y)| \leq \xi(t)|x-y|$, for each $t \in[0, T], x, y \in \mathbb{R}$, where $\xi \in L^{\frac{1}{\gamma}}\left([0, T], \mathbb{R}^{+}\right)$, $\gamma \in(0, \alpha)$.

$\left(\mathrm{H}_{4}\right)\left|J_{i}(x)-J_{i}(y)\right| \leq \eta_{i}|x-y|$, for each $x, y \in \mathbb{R}$, with constants $\eta_{i}>0, i=1,2, \ldots, m$.

Denote $\|\xi\|=\left(\int_{0}^{T}|\xi(s)|^{\frac{1}{\gamma}} d s\right)^{\gamma}$.

If

$$
\|\xi\| \Omega^{*}+\eta^{*} \Phi^{*}<1,
$$

then the impulsive fractional boundary-value problem (1.1) has a unique solution.

Proof For $x, y \in \mathrm{PC}([0, T], \mathbb{R})$ and for each $t \in[0, T]$, by Hölder's inequality, we get

$$
\begin{aligned}
& |(A x)(t)-(A y)(t)| \\
& \leq \sup _{t \in[0, T]}\left\{\frac { | b | } { | a + b | } \left[\sum_{i=1}^{m}\left|J_{i}\left(\sum_{j=1}^{i} d_{i, j} I^{\beta_{i, j}} x\left(t_{j}^{-}\right)\right)-J_{i}\left(\sum_{j=1}^{i} d_{i, j} I^{\beta_{i, j}} y\left(t_{j}^{-}\right)\right)\right|\right.\right. \\
& \left.+\frac{1}{\Gamma(\alpha)} \int_{0}^{T}(T-s)^{\alpha-1}|f(s, x(s))-f(s, y(s))| d s\right] \\
& +\sum_{i=1}^{k}\left|J_{i}\left(\sum_{j=1}^{i} d_{i, j} I^{\beta_{i, j}} x\left(t_{j}^{-}\right)\right)-J_{i}\left(\sum_{j=1}^{i} d_{i, j} I^{\beta_{i, j}} y\left(t_{j}^{-}\right)\right)\right| \\
& \left.+\frac{1}{\Gamma(\alpha)} \int_{0}^{t}(t-s)^{\alpha-1}|f(s, x(s))-f(s, y(s))| d s\right\} \\
& \leq \frac{|a+b|+|b|}{|a+b|}\left[\sum_{i=1}^{m} \eta_{i} \sum_{j=1}^{i} \frac{\left|d_{i, j}\right|}{\Gamma\left(\beta_{i, j}\right)} \int_{0}^{t_{j}}\left(t_{j}-s\right)^{\beta_{i, j}-1}|x-y| d s\right. \\
& \left.+\frac{1}{\Gamma(\alpha)} \int_{0}^{T}(T-s)^{\alpha-1} \xi(s)|x-y| d s\right] \\
& \leq\left\{\frac { | a + b | + | b | } { | a + b | } \left[\left(\sum_{i=1}^{m}\left(\eta_{i}\right)^{\frac{1}{\sigma}}\right)^{\sigma}\left(\sum_{i=1}^{m}\left(\sum_{j=1}^{i} \frac{\left|d_{i, j}\right|}{\Gamma\left(\beta_{i, j}\right)} \int_{0}^{t_{j}}\left(t_{j}-s\right)^{\beta_{i, j}-1} d s\right)^{\frac{1}{1-\sigma}}\right)^{1-\sigma}\right.\right. \\
& \left.\left.+\frac{1}{\Gamma(\alpha)}\left(\int_{0}^{T}\left((T-s)^{\alpha-1}\right)^{\frac{1}{1-\gamma}} d s\right)^{1-\gamma}\left(\int_{0}^{T}(\xi(s))^{\frac{1}{\gamma}} d s\right)^{\gamma}\right]\right\}\|x-y\| \\
& \leq\left\{\frac { | a + b | + | b | } { | a + b | } \left[\eta^{*}\left(\sum_{i=1}^{m}\left(\sum_{j=1}^{i} \frac{\left|d_{i, j}\right| t_{j}^{\beta_{i, j}}}{\Gamma\left(\beta_{i, j}+1\right)}\right)^{\frac{1}{1-\sigma}}\right)^{1-\sigma}\right.\right. \\
& \left.\left.+\frac{\|\xi\|}{\Gamma(\alpha)}\left(\frac{1-\gamma}{\alpha-\gamma}\right)^{1-\gamma} T^{\alpha-\gamma}\right]\right\}\|x-y\| \\
& =\left(\|\xi\| \Omega^{*}+\eta^{*} \Phi^{*}\right)\|x-y\| .
\end{aligned}
$$


Therefore,

$$
\|A x-A y\| \leq\left(\|\xi\| \Omega^{*}+\eta^{*} \Phi^{*}\right)\|x-y\| .
$$

It follows that $A$ is a contraction mapping. Hence Banach's fixed-point theorem implies that $A$ has a unique fixed point, which is the unique solution of the impulsive fractional boundary-value problem (1.1). This completes the proof.

\subsection{Existence result via Krasnoselskii's fixed-point theorem}

Lemma 3.1 (Krasnoselskii's fixed point theorem) [29] Let M be a closed, bounded, convex and nonempty subset of a Banach space X. Let $A, B$ be the operators such that (a) $A x+B y \in$ $M$ whenever $x, y \in M$; (b) $A$ is compact and continuous; (c) $B$ is a contraction mapping. Then there exists $z \in M$ such that $z=A z+B z$.

Theorem 3.3 Let $f:[0, T] \times \mathbb{R} \rightarrow \mathbb{R}$ be a continuous function and let $\left(\mathrm{H}_{2}\right)$ holds. In addition, we assume that:

$\left(\mathrm{H}_{5}\right) \quad|f(t, x)| \leq \mu(t), \forall(t, x) \in[0, T] \times \mathbb{R}$, and $\mu \in C\left([0, T], \mathbb{R}^{+}\right)$.

$\left(\mathrm{H}_{6}\right)$ There exists a constant $N>0$ such that $\left|J_{k}(x)\right| \leq N, \forall x \in \mathbb{R}$, for $k=1,2, \ldots, m$.

Then the impulsive fractional boundary-value problem (1.1) has at least one solution on $[0, T]$ if

$$
L_{2} \Phi<1
$$

where $\Phi$ is defined by equation (3.2).

Proof We define $\sup _{t \in[0, T]}|\mu(t)|=\|\mu\|$ and choose a suitable constant $\bar{r}$ as

$$
\bar{r} \geq\|\mu\| \Omega+\Psi,
$$

where $\Omega$ and $\Psi$ are defined by equations (3.1) and (3.3), respectively. We define the operators $\mathcal{P}$ and $\mathcal{Q}$ on $B_{\bar{r}}=\{x \in \operatorname{PC}([0, T], \mathbb{R}):\|x\| \leq \bar{r}\}$ as

$$
\begin{aligned}
& (\mathcal{P} x)(t)=-\frac{b}{(a+b) \Gamma(\alpha)} \int_{0}^{T}(T-s)^{\alpha-1} f(s, x(s)) d s+\frac{1}{\Gamma(\alpha)} \int_{0}^{t}(t-s)^{\alpha-1} f(s, x(s)) d s, \\
& (\mathcal{Q} x)(t)=\frac{c}{a+b}-\frac{b}{a+b} \sum_{i=1}^{m} J_{i}\left(\sum_{j=1}^{i} d_{i, j} I^{\beta_{i, j}} x\left(t_{j}^{-}\right)\right)+\sum_{i=1}^{k} J_{i}\left(\sum_{j=1}^{i} d_{i, j} I^{\beta_{i, j}} x\left(t_{j}^{-}\right)\right) .
\end{aligned}
$$

For $x, y \in B_{\bar{r}}$, we find that

$$
\begin{aligned}
& \|\mathcal{P} x+\mathcal{Q} y\| \\
& \quad \leq\|\mu\|\left(\frac{|a+b|+|b|}{|a+b| \Gamma(\alpha+1)}\right) T^{\alpha}+\frac{(|a+b|+|b|) m N+|c|}{|a+b|} \\
& \leq\|\mu\| \Omega+\Psi \\
& \quad \leq \bar{r} .
\end{aligned}
$$


Thus, $\mathcal{P} x+\mathcal{Q} y \in B_{\bar{r}}$. It follows from the assumption $\left(\mathrm{H}_{2}\right)$ together with (3.8) that $\mathcal{Q}$ is a contraction mapping. Continuity of $f$ implies that the operator $\mathcal{P}$ is continuous. Also, $\mathcal{P}$ is uniformly bounded on $B_{\bar{r}}$ as

$$
\|\mathcal{P} x\| \leq\|\mu\| \Omega \text {. }
$$

Now we prove the compactness of the operator $\mathcal{P}$.

We define $\sup _{(t, x) \in[0, T] \times B_{\bar{r}}}|f(t, x)|=\bar{f}<\infty, \tau_{1}, \tau_{2} \in[0, T]$ with $\tau_{1}<\tau_{2}$ and consequently we have

$$
\begin{aligned}
& \left|(\mathcal{P} x)\left(\tau_{2}\right)-(\mathcal{P} x)\left(\tau_{1}\right)\right| \\
& \quad=\frac{1}{\Gamma(\alpha)}\left|\int_{0}^{\tau_{1}}\left[\left(\tau_{2}-s\right)^{\alpha-1}-\left(\tau_{1}-s\right)^{\alpha-1}\right] f(s, x(s)) d s+\int_{\tau_{1}}^{\tau_{2}}\left(\tau_{2}-s\right)^{\alpha-1} f(s, x(s)) d s\right| \\
& \quad \leq \frac{\bar{f}}{\Gamma(\alpha+1)}\left|\tau_{2}^{\alpha}-\tau_{1}^{\alpha}\right|,
\end{aligned}
$$

which is independent of $x$ and tends to zero as $\tau_{2}-\tau_{1} \rightarrow 0$. Thus, $\mathcal{P}$ is equicontinuous. So $\mathcal{P}$ is relatively compact on $B_{\bar{r}}$. Hence, by the Arzelá-Ascoli theorem, $\mathcal{P}$ is compact on $B_{\bar{r}}$. Thus all the assumptions of Lemma 3.1 are satisfied. So the conclusion of Lemma 3.1 implies that the impulsive fractional boundary-value problem (1.1) has at least one solution on $[0, T]$. The proof is completed.

\subsection{Existence result via Leray-Schauder's Nonlinear Alternative}

Lemma 3.2 (Nonlinear alternative for single valued maps) [30] Let E be a Banach space, $C$ a closed, convex subset of $E, U$ an open subset of $C$ and $0 \in U$. Suppose that $F: \bar{U} \rightarrow C$ is a continuous, compact (that is, $F(\bar{U})$ is a relatively compact subset of $C$ ) map. Then either

(i) F has a fixed point in $\bar{U}$, or

(ii) there is a $u \in \partial U$ (the boundary of $U$ in $C$ ) and $\lambda \in(0,1)$ with $u=\lambda F(u$ ).

Theorem 3.4 Assume the following.

$\left(\mathrm{H}_{7}\right)$ There exist a continuous nondecreasing function $\psi:[0, \infty) \rightarrow(0, \infty)$ and a function $p \in L^{1}\left([0, T], \mathbb{R}^{+}\right)$such that

$$
|f(t, x)| \leq p(t) \psi(|x|) \quad \text { for each }(t, x) \in[0, T] \times \mathbb{R}
$$

$\left(\mathrm{H}_{8}\right)$ There exists a continuous nondecreasing function $\varphi:[0, \infty) \rightarrow(0, \infty)$ such that

$$
\left|J_{k}(x)\right| \leq \varphi(|x|) \quad \text { for all } x \in \mathbb{R} \text {. }
$$

$\left(\mathrm{H}_{9}\right)$ There exists a constant $M^{*}>0$ such that

$$
\frac{M^{*}}{\left(\frac{|a+b|+|b|}{|a+b|}\right) \sum_{i=1}^{m} \varphi\left(M^{*} \sum_{j=1}^{i} \frac{\mid d_{i, j} t_{j}^{\beta_{i j}}}{\Gamma\left(\beta_{i, j}+1\right)}\right)+\psi\left(M^{*}\right)\|p\|_{L_{1}} \Omega+\frac{|c|}{|a+b|}}>1 .
$$

Then the impulsive fractional boundary-value problem (1.1) has at least one solution on $[0, T]$. 
Proof We show that $A$ maps bounded sets (balls) into bounded sets in $\mathrm{PC}([0, T], \mathbb{R})$. For a positive number $r$, let $B_{r}=\{x \in C([0, T], \mathbb{R}):\|x\| \leq r\}$ be a bounded ball in $\mathrm{PC}([0, T], \mathbb{R})$. Then for $t \in[0, T]$ we have

$$
\begin{aligned}
|A x(t)| & \leq \frac{|b|}{|a+b|}\left[\sum_{i=1}^{m}\left|J_{i}\left(\sum_{j=1}^{i} d_{i, j} I^{\beta_{i, j}} x\left(t_{j}^{-}\right)\right)\right|+\frac{1}{\Gamma(\alpha)} \int_{0}^{T}(T-s)^{\alpha-1}|f(s, x(s))| d s\right] \\
& +\sum_{i=1}^{k}\left|J_{i}\left(\sum_{j=1}^{i} d_{i, j} I^{\beta_{i, j}} x\left(t_{j}^{-}\right)\right)\right|+\frac{1}{\Gamma(\alpha)} \int_{0}^{t}(t-s)^{\alpha-1}|f(s, x(s))| d s+\frac{|c|}{|a+b|} \\
\leq & \frac{|a+b|+|b|}{|a+b|}\left[\sum_{i=1}^{m}\left|J_{i}\left(\sum_{j=1}^{i} d_{i, j} I^{\beta_{i, j}} x\left(t_{j}^{-}\right)\right)\right|+\frac{1}{\Gamma(\alpha)} \int_{0}^{T}(T-s)^{\alpha-1}|f(s, x(s))| d s\right] \\
& +\frac{|c|}{|a+b|} \\
\leq & \frac{|a+b|+|b|}{|a+b|}\left[\sum_{i=1}^{m} \varphi\left(\left|\sum_{j=1}^{i} d_{i, j} \beta^{\beta_{i, j}} x\left(t_{j}^{-}\right)\right|\right)+\frac{\psi(\|x\|)}{\Gamma(\alpha)} \int_{0}^{T}(T-s)^{\alpha-1} p(s) d s\right] \\
& +\frac{|c|}{|a+b|} \\
\leq & \frac{|a+b|+|b|}{|a+b|}\left[\sum_{i=1}^{m} \varphi\left(\sum_{j=1}^{i} \frac{\left|d_{i, j}\right| t_{j}^{\beta_{i, j}}}{\Gamma\left(\beta_{i, j}+1\right)}\|x\|\right)+\frac{\psi(\|x\|)\|p\|_{L_{1}}}{\Gamma(\alpha+1)} T^{\alpha}\right]+\frac{|c|}{|a+b|} .
\end{aligned}
$$

Consequently

$$
\|A x\| \leq \frac{|a+b|+|b|}{|a+b|} \sum_{i=1}^{m} \varphi\left(\sum_{j=1}^{i} \frac{\left|d_{i, j}\right| t_{j}^{\beta_{i, j}}}{\Gamma\left(\beta_{i, j}+1\right)} r\right)+\psi(r)\|p\|_{L_{1}} \Omega+\frac{|c|}{|a+b|} .
$$

Next we show that $A$ maps bounded sets into equicontinuous sets of $\mathrm{PC}([0, T], \mathbb{R})$. Let $\sup _{(t, x) \in[0, T] \times B_{r}}|f(t, x)|=f^{*}<\infty, \tau_{1}, \tau_{2} \in[0, T]$ with $\tau_{1} \in\left(t_{u}, t_{u+1}\right], \tau_{2} \in\left(t_{v}, t_{v+1}\right], u \leq v, u, v \in$ $\{1,2, \ldots, m\}$ and $x \in B_{r}$. Then we have

$$
\begin{aligned}
\left|(A x)\left(\tau_{2}\right)-(A x)\left(\tau_{1}\right)\right| \leq & \frac{1}{\Gamma(\alpha)} \mid \int_{0}^{\tau_{1}}\left[\left(\tau_{2}-s\right)^{\alpha-1}-\left(\tau_{1}-s\right)^{\alpha-1}\right] f(s, x(s)) d s \\
& +\int_{\tau_{1}}^{\tau_{2}}\left(\tau_{2}-s\right)^{\alpha-1} f(s, x(s)) d s\left|+\sum_{i=u+1}^{v}\right| J_{i}\left(\sum_{j=1}^{i} d_{i, j} I^{\beta_{i, j}} x\left(t_{j}^{-}\right)\right) \mid \\
\leq & \frac{f^{*}}{\Gamma(\alpha+1)}\left|\tau_{2}^{\alpha}-\tau_{1}^{\alpha}\right|+\sum_{i=u+1}^{v}\left|J_{i}\left(\sum_{j=1}^{i} d_{i, j} I^{\beta_{i, j}} x\left(t_{j}^{-}\right)\right)\right|
\end{aligned}
$$

Obviously the right-hand side of the above inequality tends to zero independently of $x \in B_{r}$ as $\tau_{2}-\tau_{1} \rightarrow 0$. As $A$ satisfies the above assumptions, therefore it follows by the ArzeláAscoli theorem that $A: \mathrm{PC}([0, T], \mathbb{R}) \rightarrow \mathrm{PC}([0, T], \mathbb{R})$ is completely continuous. 
Let $x$ be a solution. Then, for $t \in[0, T]$, and following the similar computations as in the first step, we have

$$
\|x\| \leq \frac{|a+b|+|b|}{|a+b|} \sum_{i=1}^{m} \varphi\left(\sum_{j=1}^{i} \frac{\left|d_{i, j}\right| t_{j}^{\beta_{i, j}}}{\Gamma\left(\beta_{i, j}+1\right)}\|x\|\right)+\psi(\|x\|)\|p\|_{L_{1}} \Omega+\frac{|c|}{|a+b|} .
$$

Consequently, we have

$$
\frac{\|x\|}{\left(\frac{|a+b|+|b|}{|a+b|}\right) \sum_{i=1}^{m} \varphi\left(\sum_{j=1}^{i} \frac{\left|d_{i, j}\right| t_{j} t_{i j}}{\Gamma\left(\beta_{i, j}+1\right)}\|x\|\right)+\psi(\|x\|)\|p\|_{L_{1}} \Omega+\frac{|c|}{|a+b|}} \leq 1 .
$$

In view of $\left(\mathrm{H}_{9}\right)$, there exists $M^{*}$ such that $\|x\| \neq M^{*}$. Let us set

$$
U=\left\{x \in \mathrm{PC}([0, T], \mathbb{R}):\|x\|<M^{*}\right\} .
$$

Note that the operator $A: \bar{U} \rightarrow \mathrm{PC}([0, T], \mathbb{R})$ is continuous and completely continuous. From the choice of $U$, there is no $x \in \partial U$ such that $x=\lambda A x$ for some $\lambda \in(0,1)$. Consequently, by the nonlinear alternative of Leray-Schauder type (Lemma 3.2), we deduce that $A$ has a fixed point $x \in \bar{U}$ which is a solution of the problem (1.1). This completes the proof.

\subsection{Existence result via Leray-Schauder degree}

Theorem 3.5 Assume the following.

$\left(\mathrm{H}_{10}\right)$ There exist constants $0 \leq \kappa<\Omega^{-1}$ and $M>0$ such that

$$
|f(t, x)| \leq \kappa|x|+M \quad \text { for all }(t, x) \in[0, T] \times \mathbb{R} .
$$

$\left(\mathrm{H}_{11}\right)$ There exist constants $0 \leq \gamma<(1-\kappa \Omega) \Phi^{-1}$ and $N>0$ such that

$$
\left|J_{k}(x)\right| \leq \gamma|x|+N \quad \text { for all } x \in \mathbb{R}
$$

where $\Omega$ and $\Phi$ are given by equations (3.1) and (3.2), respectively.

Then the impulsive fractional boundary-value problem (1.1) has at least one solution on $[0, T]$.

Proof We define an operator $A: \mathrm{PC}([0, T], \mathbb{R}) \rightarrow \mathrm{PC}([0, T], \mathbb{R})$ as in equation (2.4) and consider the fixed-point problem

$$
x=A x .
$$

We are going to prove that there exists a fixed point $x \in \mathrm{PC}([0, T], \mathbb{R})$ satisfying equation (3.9). It is sufficient to show that $A: \bar{B}_{R} \rightarrow \mathrm{PC}([0, T], \mathbb{R})$ satisfies

$$
x \neq \lambda A x, \quad \forall x \in \partial B_{R}, \forall \lambda \in[0,1],
$$


where $B_{R}=\left\{x \in \operatorname{PC}([0, T], \mathbb{R}): \max _{t \in[0, T]}|x(t)|<R, R>0\right\}$. We define

$$
H(\lambda, x)=\lambda A x, \quad x \in \operatorname{PC}([0, T], \mathbb{R}), \lambda \in[0,1] .
$$

As shown in Theorem 3.4, we find that the operator $A$ is continuous, uniformly bounded, and equicontinuous. Then, by the Arzelá-Ascoli theorem, a continuous map $h_{\lambda}$ defined by $h_{\lambda}(x)=x-H(\lambda, x)=x-\lambda A x$ is completely continuous. If equation (3.10) is true, then the following Leray-Schauder degrees are well defined and by the homotopy invariance of topological degree, it follows that

$$
\begin{aligned}
\operatorname{deg}\left(h_{\lambda}, B_{R}, 0\right) & =\operatorname{deg}\left(I-\lambda A, B_{R}, 0\right)=\operatorname{deg}\left(h_{1}, B_{R}, 0\right) \\
& =\operatorname{deg}\left(h_{0}, B_{R}, 0\right)=\operatorname{deg}\left(I, B_{R}, 0\right)=1 \neq 0, \quad 0 \in B_{R},
\end{aligned}
$$

where $I$ denotes the identity operator. By the nonzero property of the Leray-Schauder degree, $h_{1}(x)=x-A x=0$ for at least one $x \in B_{R}$. In order to prove equation (3.10), we assume that $x=\lambda A x$ for some $\lambda \in[0,1]$. Then

$$
\begin{aligned}
|A x(t)| & \leq \sup _{t \in[0, T]}\left\{\frac{|b|}{|a+b|}\left[\sum_{i=1}^{m}\left|J_{i}\left(\sum_{j=1}^{i} d_{i, j} I^{\beta_{i, j}} x\left(t_{j}^{-}\right)\right)\right|+\frac{1}{\Gamma(\alpha)} \int_{0}^{T}(T-s)^{\alpha-1}|f(s, x(s))| d s\right]\right. \\
& \left.+\sum_{i=1}^{k}\left|J_{i}\left(\sum_{j=1}^{i} d_{i, j} \beta_{i, j}^{\beta_{i, j}} x\left(t_{j}^{-}\right)\right)\right|+\frac{1}{\Gamma(\alpha)} \int_{0}^{t}(t-s)^{\alpha-1}|f(s, x(s))| d s+\frac{|c|}{|a+b|}\right\} \\
\leq & \frac{|a+b|+|b|}{|a+b|}\left[\sum_{i=1}^{m}\left|J_{i}\left(\sum_{j=1}^{i} d_{i, j} I^{\beta_{i, j}} x\left(t_{j}^{-}\right)\right)\right|+\frac{1}{\Gamma(\alpha)} \int_{0}^{T}(T-s)^{\alpha-1}|f(s, x(s))| d s\right] \\
& +\frac{|c|}{|a+b|} \\
\leq & \frac{|a+b|+|b|}{|a+b|}\left[\sum_{i=1}^{m}\left(\gamma\left|\sum_{j=1}^{i} d_{i, j} \beta^{\beta_{i, j}} x\left(t_{j}^{-}\right)\right|+N\right)+\frac{\kappa|x|+M}{\Gamma(\alpha)} \int_{0}^{T}(T-s)^{\alpha-1} d s\right] \\
& +\frac{|c|}{|a+b|} \\
\leq & \frac{|a+b|+|b|}{|a+b|}\left[\left(\gamma\|x\| \sum_{i=1}^{m} \sum_{j=1}^{i} \frac{\left|d_{i, j}\right| t_{j}^{\beta_{i, j}}}{\Gamma\left(\beta_{i, j}+1\right)}+m N\right)+\frac{\kappa\|x\|+M}{\Gamma(\alpha+1)} T^{\alpha}\right]+\frac{|c|}{|a+b|} \\
\leq & (\kappa\|x\|+M) \Omega+\gamma\|x\| \Phi+\Psi .
\end{aligned}
$$

Computing directly for $\|x\|=\sup _{t \in[0, T]}|x(t)|$, we have

$$
\|x\| \leq \frac{M \Omega+\Psi}{1-\kappa \Omega-\gamma \Phi} .
$$

If $R=\frac{M \Omega+\Psi}{1-\kappa \Omega-\gamma \Phi}+1$, inequality (3.10) holds. This completes the proof. 


\section{Examples}

In this section we give examples to illustrate our results.

Example 4.1 Consider the following impulsive fractional boundary-value problem:

$$
\begin{aligned}
& { }^{c} D^{\frac{2}{3}} x(t)=\frac{\sin \pi t}{(t+1)} \cdot \frac{|x(t)|}{4+2|x(t)|}, \quad t \in\left(0, \frac{3}{4}\right), t \neq \frac{1}{4}, \frac{1}{2}, \\
& \Delta x\left(\frac{1}{4}\right)=J_{1}\left(\frac{1}{2} \int_{0}^{\frac{1}{4}} \frac{(t-s)^{-\frac{3}{5}}}{\Gamma\left(\frac{2}{5}\right)} x(s) d s\right), \\
& \Delta x\left(\frac{1}{2}\right)=J_{2}\left(\frac{1}{3} \int_{0}^{\frac{1}{4}} \frac{(t-s)^{-\frac{2}{3}}}{\Gamma\left(\frac{1}{3}\right)} x(s) d s+\frac{2}{3} \int_{0}^{\frac{1}{2}} \frac{(t-s)^{-\frac{3}{4}}}{\Gamma\left(\frac{1}{4}\right)} x(s) d s\right), \\
& 5 x(0)+4 x\left(\frac{3}{4}\right)=2,
\end{aligned}
$$

where $J_{1}(u)=|u| /(3+|u|), J_{2}(u)=|u| /(4+|u|)$.

Set $\alpha=2 / 3, T=3 / 4, d_{1,1}=1 / 2, d_{2,1}=1 / 3, d_{2,2}=2 / 3, \beta_{1,1}=2 / 5, \beta_{2,1}=1 / 3, \beta_{2,2}=1 / 4, a=5$, $b=4$ and $c=2$.

Since $|f(t, x)-f(t, y)| \leq(1 / 4)|x-y|$ and $\left|J_{k}(u)-J_{k}(v)\right| \leq(1 / 3)|u-v|$ for $k=1,2$, then $\left(\mathrm{H}_{1}\right)$ and $\left(\mathrm{H}_{2}\right)$ are satisfied with $L_{1}=1 / 4$ and $L_{2}=1 / 3$. We can show that

$$
L_{1} \Omega+L_{2} \Phi \approx 0.9581219078<1
$$

Hence, by Theorem 3.1, the boundary-value problem (4.1)-(4.3) has a unique solution on $[0,3 / 4]$.

Example 4.2 Consider the following impulsive fractional boundary-value problem:

$$
\begin{aligned}
& { }^{c} D^{\frac{4}{5}} x(t)=\frac{e^{t}}{2\left(e^{t}+1\right)} \cdot \frac{|x(t)|}{2+|x(t)|}, \quad t \in\left(0, \frac{2}{3}\right), t \neq \frac{1}{4}, \frac{1}{3}, \\
& \begin{array}{ll}
\Delta x\left(\frac{1}{4}\right) & =J_{1}\left(\frac{2}{5} \int_{0}^{\frac{1}{4}} \frac{(t-s)^{-\frac{3}{4}}}{\Gamma\left(\frac{1}{4}\right)} x(s) d s\right), \\
\Delta x\left(\frac{1}{3}\right) & =J_{2}\left(\frac{2}{3} \int_{0}^{\frac{1}{4}} \frac{(t-s)^{-\frac{3}{5}}}{\Gamma\left(\frac{2}{5}\right)} x(s) d s+\frac{1}{3} \int_{0}^{\frac{1}{3}} \frac{(t-s)^{-\frac{2}{5}}}{\Gamma\left(\frac{3}{5}\right)} x(s) d s\right), \\
5 x(0)+4 x\left(\frac{2}{3}\right)=5,
\end{array}
\end{aligned}
$$

where $J_{1}(u)=(u / 3)-5, J_{2}(u)=2|u| /(4+3|u|)$.

Set $\alpha=4 / 5, T=2 / 3, d_{1,1}=2 / 5, d_{2,1}=2 / 3, d_{2,2}=1 / 3, \beta_{1,1}=1 / 4, \beta_{2,1}=2 / 5, \beta_{2,2}=3 / 5, a=5$, $b=4$ and $c=5$.

Since $|f(t, x)-f(t, y)| \leq\left[e^{t} /\left(4\left(e^{t}+1\right)\right)\right]|x-y|,\left|J_{1}(u)-J_{1}(v)\right| \leq(1 / 3)|u-v|$ and $\mid J_{2}(u)-$ $J_{2}(v)|\leq(1 / 2)| u-v \mid$, then $\left(\mathrm{H}_{3}\right)$ and $\left(\mathrm{H}_{4}\right)$ are satisfied with $\xi(t)=e^{t} /\left(4\left(e^{t}+1\right)\right), \eta_{1}=1 / 3$, 
$\eta_{2}=1 / 2, \gamma=3 / 5$ and $\sigma=2 / 3$. We can show that

$$
\|\xi\| \Omega^{*}+\eta^{*} \Phi^{*} \approx 0.8454119129<1 .
$$

Hence, by Theorem 3.2, the boundary-value problem (4.4)-(4.6) has a unique solution on $[0,2 / 3]$.

Example 4.3 Consider the following impulsive fractional boundary-value problem:

$$
\begin{aligned}
& { }^{c} D^{\frac{3}{5}} x(t)=\frac{\sin \pi x}{2 \pi^{2}+\sin ^{2} \pi x}+\frac{1+\sin \pi t}{2 \pi}, \quad t \in\left(0, \frac{1}{2}\right), t \neq \frac{1}{4}, \frac{1}{3}, \\
& \begin{aligned}
& \Delta x\left(\frac{1}{4}\right)=J_{1}\left(\frac{1}{2} \int_{0}^{\frac{1}{4}} \frac{(t-s)^{-\frac{3}{5}}}{\Gamma\left(\frac{2}{5}\right)} x(s) d s\right), \\
& \Delta x\left(\frac{1}{3}\right)=J_{2}\left(\frac{1}{3} \int_{0}^{\frac{1}{4}} \frac{(t-s)^{-\frac{2}{5}}}{\Gamma\left(\frac{3}{5}\right)} x(s) d s+\int_{0}^{\frac{1}{3}} \frac{(t-s)^{-\frac{2}{3}}}{\Gamma\left(\frac{1}{3}\right)} x(s) d s\right), \\
& 4 x(0)+3 x\left(\frac{1}{2}\right)=3,
\end{aligned}
\end{aligned}
$$

where $J_{1}(u)=(\sin \pi u) /\left(2 \pi^{2}\right), J_{2}(u)=2 u /\left(3 \pi+u^{2}\right)$.

Set $\alpha=3 / 5, T=1 / 2, d_{1,1}=1 / 2, d_{2,1}=1 / 3, d_{2,2}=1, \beta_{1,1}=2 / 5, \beta_{2,1}=3 / 5, \beta_{2,2}=1 / 3, a=4$, $b=3, c=3$ and $f(t, x)=\left((\sin \pi x) /\left(2 \pi^{2}+\sin ^{2} \pi x\right)\right)+((1+\sin \pi t) /(2 \pi))$.

It is easy to see that $\Omega=1.054828718$. Clearly,

$$
\begin{aligned}
& |f(t, x)|=\left|\frac{\sin \pi x}{2 \pi^{2}+\sin ^{2} \pi x}+\frac{1+\sin \pi t}{2 \pi}\right| \leq(1+\sin \pi t)\left(\frac{|x|+1}{2 \pi}\right), \\
& \left|J_{1}(u)\right|=\frac{|\sin \pi u|}{2 \pi^{2}} \leq \frac{|u|}{2 \pi},
\end{aligned}
$$

and

$$
\left|J_{2}(u)\right|=\left|\frac{2 u}{3 \pi+u^{2}}\right| \leq \frac{2|u|}{3 \pi} .
$$

Choosing $p(t)=1+\sin \pi t, \psi(|x|)=(|x|+1) /(2 \pi)$ and $\varphi(|u|)=2|u| / 3 \pi$, we obtain

$$
\frac{M^{*}}{0.5659502780+0.5201099305 M^{*}}>1
$$

which implies that $M^{*}>1.179333172$. Hence, by Theorem 3.4, the boundary-value problem (4.7)-(4.9) has at least one solution on [0,1/2].

Example 4.4 Consider the following impulsive fractional boundary-value problem:

$$
\begin{aligned}
{ }^{c} D^{\frac{3}{4}} x(t) & =\frac{t \sin \pi x}{2(t+5)^{2}\left(x^{2}+1\right)}+\frac{2 t}{(t+2)^{2}}, \quad t \in\left(0, \frac{4}{3}\right), t \neq \frac{1}{4}, \frac{2}{3}, \frac{3}{4}, \\
\Delta x\left(\frac{1}{4}\right) & =J_{1}\left(\frac{1}{3} \int_{0}^{\frac{1}{4}} \frac{(t-s)^{-\frac{1}{2}}}{\Gamma\left(\frac{1}{2}\right)} x(s) d s\right),
\end{aligned}
$$




$$
\begin{aligned}
& \Delta x\left(\frac{2}{3}\right)= J_{2}\left(\frac{1}{2} \int_{0}^{\frac{1}{4}} \frac{(t-s)^{-\frac{2}{3}}}{\Gamma\left(\frac{1}{3}\right)} x(s) d s+\frac{1}{4} \int_{0}^{\frac{2}{3}} \frac{(t-s)^{-\frac{1}{3}}}{\Gamma\left(\frac{2}{3}\right)} x(s) d s\right), \\
& \Delta x\left(\frac{3}{4}\right)= J_{3}\left(\frac{1}{3} \int_{0}^{\frac{1}{4}} \frac{(t-s)^{-\frac{3}{4}}}{\Gamma\left(\frac{1}{4}\right)} x(s) d s+\frac{2}{3} \int_{0}^{\frac{2}{3}} \frac{(t-s)^{-\frac{1}{2}}}{\Gamma\left(\frac{1}{2}\right)} x(s) d s\right. \\
&\left.+\frac{1}{4} \int_{0}^{\frac{3}{4}} \frac{(t-s)^{-\frac{1}{4}}}{\Gamma\left(\frac{3}{4}\right)} x(s) d s\right), \\
& 4 x(0)+5 x\left(\frac{4}{3}\right)=3,
\end{aligned}
$$

where $J_{1}(u)=\left(u /\left(6+u^{2}\right)\right)+1, J_{2}(u)=\left(u /\left(6+\sin ^{2} u\right)\right)+(1 / 3), J_{3}(u)=((\sin \pi u) /(7 \pi))+(1 / 2)$.

Set $\alpha=3 / 4, T=4 / 3, d_{1,1}=1 / 3, d_{2,1}=1 / 2, d_{2,2}=1 / 4, d_{3,1}=1 / 3, d_{3,2}=2 / 3, d_{3,3}=1 / 4$, $\beta_{1,1}=1 / 2, \beta_{2,1}=1 / 3, \beta_{2,2}=2 / 3, \beta_{3,1}=1 / 4, \beta_{3,2}=1 / 2, \beta_{3,3}=3 / 4, a=4, b=5$ and $c=3$.

Since $|f(t, x)| \leq(1 / 6)|x|+1,\left|J_{k}(x)\right| \leq(1 / 6)|x|+1$ for $k=1,2,3$, then $\left(\mathrm{H}_{10}\right)$ and $\left(\mathrm{H}_{11}\right)$ are satisfied with $\kappa=1 / 6, \gamma=1 / 6, M=1$ and $N=1$. We have

$$
\kappa=0.1666666667<0.4761628182=\Omega^{-1}
$$

and

$$
\lambda=0.1666666667<0.2080746384=(1-\kappa \Omega) \Phi^{-1} .
$$

Hence, by Theorem 3.5, the boundary value-problem (4.10)-(4.12) has at least one solution on $[0,4 / 3]$.

\section{Competing interests}

The authors declare that they have no competing interests.

\section{Authors' contributions}

All authors contributed equally in this article. They read and approved the final manuscript.

\section{Author details}

1 Department of Mathematics, Faculty of Applied Science, King Mongkut's University of Technology North Bangkok, Bangkok, Thailand. ${ }^{2}$ Department of Mathematics, University of loannina, Ioannina, 451 10, Greece.

\section{Authors' information}

The third author is a Member of Nonlinear Analysis and Applied Mathematics (NAAM)-Research Group at King Abdulaziz University, Jeddah, Saudi Arabia.

\section{Acknowledgements}

We would like to thank the reviewers for their valuable comments and suggestions on the manuscript. This research of CT and JT is supported by King Mongkut's University of Technology North Bangkok, Thailand.

Received: 25 July 2013 Accepted: 17 December 2013 Published: 15 Jan 2014

\section{References}

1. Guezane-Lakoud, A, Khaldi, R: Positive solution to a higher order fractional boundary value problem with fractional integral condition. Rom. J. Math. Comput. Sci. 2, 41-54 (2012)

2. Kaufmann, E: Existence and nonexistence of positive solutions for a nonlinear fractional boundary value problem. Discrete Contin. Dyn. Syst. 2009, suppl., 416-423 (2009)

3. Wang, J, Xiang, H, Liu, Z: Positive solution to nonzero boundary values problem for a coupled system of nonlinear fractional differential equations. Int. J. Differ. Equ. 2010, Article ID 186928 (2010)

4. Benchohra, M, Henderson, J, Ntouyas, SK, Ouahab, A: Existence results for fractional order functional differential equations with infinite delay. J. Math. Anal. Appl. 338, 1340-1350 (2008)

5. Bai, Z: On positive solutions of a nonlocal fractional boundary value problem. Nonlinear Anal. 72, 916-924 (2010)

6. Sudsutad, W, Tariboon, J: Boundary value problems for fractional differential equations with three-point fractional integral boundary conditions. Adv. Differ. Equ. 2012, 93 (2012) 
7. Ntouyas, SK: Existence results for nonlocal boundary value problems for fractional differential equations and inclusions with fractional integral boundary conditions. Discuss. Math., Differ. Incl. Control Optim. 33, 17-39 (2013)

8. Ntouyas, SK: Boundary value problems for nonlinear fractional differential equations and inclusions with nonlocal and fractional integral boundary conditions. Opusc. Math. 33, 117-138 (2013)

9. Guezane-Lakoud, A, Khaldi, R: Solvability of a fractional boundary value problem with fractional integral condition. Nonlinear Anal. 75, 2692-2700 (2012)

10. Ahmad, B, Ntouyas, SK, Assolani, A: Caputo type fractional differential equations with nonlocal Riemann-Liouville integral boundary conditions. J. Appl. Math. Comput. 41, 339-350 (2013)

11. Baleanu, D, Mustafa, OG, Agarwal, RP: An existence result for a superlinear fractional differential equation. Appl. Math. Lett. 23, 1129-1132 (2010)

12. Debbouche, A, Baleanu, D, Agarwal, RP: Nonlocal nonlinear integrodifferential equations of fractional orders. Bound. Value Probl. 2012, 78 (2012)

13. Nyamoradi, N, Baleanu, D, Agarwal, RP: On a multipoint boundary value problem for a fractional order differential inclusion on an infinite interval. Adv. Math. Phys. 2013, Article ID 823961 (2013)

14. Agarwal, RP, Ahmad, B: Existence of solutions for impulsive anti-periodic boundary value problems of fractional semilinear evolution equations. Dyn. Contin. Discrete Impuls. Syst., Ser. A Math. Anal. 18, 535-544 (2011)

15. Samoilenko, AM, Perestyuk, NA: Impulsive Differential Equations. World Scientific, Singapore (1995)

16. Benchohra, M, Henderson, J, Ntouyas, SK: Impulsive Differential Equations and Inclusions. Hindawi Publishing, New York (2006)

17. Ahmad, B, Nieto, JJ: Existence of solutions for impulsive anti-periodic boundary value problems of fractional order. Taiwan. J. Math. 15, 981-993 (2011)

18. Ahmad, B, Wang, G: A study of an impulsive four-point nonlocal boundary value problem of nonlinear fractional differential equations. Comput. Math. Appl. 62, 1341-1349 (2011)

19. Ahmad, B, Sivasundaram, S: Existence results for nonlinear impulsive hybrid boundary value problems involving fractional differential equations. Nonlinear Anal. Hybrid Syst. 3, 251-258 (2009)

20. Ahmad, B, Sivasundaram, S: Existence of solutions for impulsive integral boundary value problems of fractional order. Nonlinear Anal. Hybrid Syst. 4, 134-141 (2010)

21. Tian, Y, Bai, Z: Existence results for the three-point impulsive boundary value problem involving fractional differential equations. Comput. Math. Appl. 59, 2601-2609 (2010)

22. Wang, G, Ahmad, B, Zhang, L: Impulsive anti-periodic boundary value problem for nonlinear differential equations of fractional order. Nonlinear Anal. 74, $792-804$ (2011)

23. Wang, G, Ahmad, B, Zhang, L: Some existence results for impulsive nonlinear fractional differential equations with mixed boundary conditions. Comput. Math. Appl. 62, 1389-1397 (2011)

24. Zhang, $X$, Huang, $X, L i u, Z$ : The existence and uniqueness of mild solutions for impulsive fractional equations with nonlocal conditions and infinite delay. Nonlinear Anal. Hybrid Syst. 4, 775-781 (2010)

25. Miller, KS, Ross, B: An Introduction to the Fractional Calculus and Differential Equations. Wiley, New York (1993)

26. Samko, SG, Kilbas, AA, Marichev, Ol: Fractional Integrals and Derivatives, Theory and Applications. Gordon \& Breach, Yverdon (1993)

27. Podlubny, I: Fractional Differential Equations. Academic Press, San Diego (1999)

28. Fečkan, M, Zhou, Y, Wang, J: On the concept and existence of solution for impulsive fractional differential equations. Commun. Nonlinear Sci. Numer. Simul. 17, 3050-3060 (2012)

29. Krasnoselskii, MA: Two remarks on the method of successive approximations. Usp. Mat. Nauk 10, 123-127 (1955)

30. Granas, A, Dugundji, J: Fixed Point Theory. Springer, New York (2003)

10.1186/1687-2770-2014-17

Cite this article as: Thaiprayoon et al.: Impulsive fractional boundary-value problems with fractional integral jump conditions. Boundary Value Problems 2014, 2014:17

\section{Submit your manuscript to a SpringerOpen ${ }^{\circ}$ journal and benefit from:}

- Convenient online submission

- Rigorous peer review

- Immediate publication on acceptance

- Open access: articles freely available online

- High visibility within the field

- Retaining the copyright to your article 